\title{
Seminare 2005
}

Obwohl rund $40 \%$ aller Ärztinnen und Ärzte früher oder später eine eigene Arztpraxis führen und nebst dem medizinischen Know-how auch jenes eines Unternehmers innehaben sollten, wird im Verlauf des Medizinstudiums die Thematik der Unternehmensführung nie oder nur kaum fokussiert. Die FMH Services, welche sich um die betriebswirtschaftlichen Angelegenheiten der FMH-Mitglieder kümmern, schliessen diese Lücke und bieten dazu spezifische Seminare an. In diesen Seminaren werden den Ärztinnen und Ärzten Grundlagen vermittelt für den Einstieg in die eigene Arzt-/Gruppenpraxis, deren Führung und deren optimale Übergabe an einen/eine Nachfolger/in. Die Ärztinnen und Ärzte werden auf den Umgang und die Zusammenarbeit mit Behörden sowie Fachspezialisten, wie Treuhänder, Versicherungs- und Finanzberater, Kreditberater bei Banken, Juristen, Architekten usw., vorbereitet. Die einzelnen Themen werden von ausgewiesenen Experten der FMH und der FMH Services referiert.

\section{Praxiseröffnung}

\section{Teilnehmende}

Das Seminar richtet sich an Ärztinnen und Ärzte, welche vor einer Praxiseröffnung oder einer Praxisübernahme stehen.

\section{Themen}

Juristische Aspekte (Praxisbewilligung, Zulassung zur Sozialversicherung, Vertragswesen), TARMED (Überblick über Tarifsystem und dessen Anwendung im Hinblick auf die Budgetierung), Praxiseinrichtung (Raumplanung, Inneneinrichtung, Kostenberechnung), Praxisadministration (Leistungserfassungs- und Abrechnungssysteme, Praxismarketing), Finanzierung einer Arztpraxis (Businessplan, Kreditarten, Absicherungsmöglichkeiten), Versicherungen (Personen- und Sachversicherungen rund um die Arztpraxis).

\section{Unterlagen}

Die Seminarteilnehmer/innen erhalten einen Seminarordner, welcher, aufbauend auf einer Checkliste für die Praxiseröffnung, Basisinformationen zu den behandelten und weiteren individuell wichtigen Themen beinhaltet.

\section{Kosten/Sponsoren}

Das Seminar ist für FMH-Mitglieder gratis. Die Kosten werden von folgenden Sponsoren gedeckt: Versicherung der Schweizer Ärzte, Schweizerische Ärzte-Krankenkasse, Druckerei SchmidMogelsberg, Labornetz daCapo, TMH Informatik $\mathrm{GmbH}$ und EMH Schweizerischer Ärzteverlag.

\section{Daten}

\begin{tabular}{llll} 
Nr. 02 & $\begin{array}{l}\text { Donnerstag, } \\
\text { Bern }\end{array}$ & $\begin{array}{l}\text { 2. Juni 2005 } \\
\text { 9.00-16.30 Uhr }\end{array}$ & $\begin{array}{l}\text { Hotel } \\
\text { Bern }\end{array}$ \\
\hline Nr. 03 & $\begin{array}{l}\text { Donnerstag, } \\
\text { Zürich }\end{array}$ & $\begin{array}{l}\text { 1. September 2005 } \\
\text { 9.00-16.30 Uhr }\end{array}$ & $\begin{array}{l}\text { FMT } \\
\text { Zürich }\end{array}$
\end{tabular}

\section{Anmeldung}

Sie können sich bei nachfolgend aufgeführter Adresse (FMH Consulting Services) oder via Internet unter www.fmhservices.ch einschreiben.

\section{Praxisübergabe/-übernahme}

\section{Teilnehmende}

Das Seminar richtet sich an zukünftige Praxisübergeber/innen sowie Praxisübernehmer/innen. Hinweis: Für Praxisübergeber/innen lohnt es sich, sich bereits frühzeitig (5-10 Jahre) mit diesem Thema auseinanderzusetzen.

\section{Themen}

Juristische Aspekte (Übergabe der Krankengeschichten, Abschluss Praxisübernahmevertrag, Mietvertrag), Ehe- und Erbrecht (Ehegüterstand, Erbschaftsfolge/-planung), Versicherungen (Übergabe/Übernahme und Abschluss/Auflösung von Versicherungsverträgen, Vorsorgeplanung), Bewertung einer Arztpraxis (Berechnung Inventarwert und Goodwill als Verhandlungsbasis), Steuern (Liquidations- und Grundstückgewinnsteuer, Steuerfolgen bei Aufnahme der Selbständigerwerbstätigkeit, Bestimmung des optimalen Übergabezeitpunkts).

\section{Unterlagen}

Die Seminarteilnehmer/innen erhalten einen Seminarordner mit Unterlagen zu den behandelten Themen. 


\section{Kosten/Sponsoren}

Das Seminar ist für FMH-Mitglieder gratis. Die Kosten werden von folgenden Sponsoren gedeckt: Versicherung der Schweizer Ärzte, Schweizerische Ärzte-Krankenkasse, Medizinische Analytik AG, Upsamedica GmbH und EMH Schweizerischer Ärzteverlag.

\section{Daten}

\begin{tabular}{llll} 
Nr. 06 & Donnerstag, 9. Juni 2005 & Hotel \\
& Bern & $9.30-16.30$ Uhr & Bern \\
\hline Nr. 07 & $\begin{array}{l}\text { Donnerstag, 8. September 2005 } \\
\text { Zürich }\end{array}$ & FMT \\
& & & Zü30-16.30 Uhr
\end{tabular}

\section{Anmeldung}

Sie können sich bei nachfolgend aufgeführter Adresse (FMH Consulting Services) oder via Internet unter www.fmhservices.ch einschreiben.

\section{Buchhaltung und Steuern}

\section{Teilnehmende}

Das Seminar richtet sich an Praxiseröffner/innen, Praxisübernehmer/innen sowie an bereits praxistätige Ärztinnen und Ärzte.

\section{Themen}

Im Buchhaltungsteil erfahren die Seminarteilnehmer/innen, welche Massnahmen vor der Praxiseröffnung getroffen werden müssen und welches deren organisatorische Voraussetzungen sind. Basierend auf dem Standardkontenplan für Ärzte wird die Führung einer doppelten Buchhaltung aufgezeigt. Zudem erhalten sie Einblick in eine EDV-unterstützte Buchführungslösung. Im Steuerteil werden die Methoden der Steuerplanung aus versicherungstechnischer sowie aus treuhänderischer Sicht aufgezeigt.

\section{Unterlagen}

Die Seminarteilnehmer/innen erhalten einen Seminarordner mit Unterlagen zu den behandelten Themen.

\section{Kosten/Sponsor}

Das Seminar ist für FMH-Mitglieder gratis. Die Kosten werden von folgendem Sponsor gedeckt: EMH Schweizerischer Ärzteverlag

\section{Daten}

\begin{tabular}{|llrl}
\multirow{2}{*}{ Nr. 10 } & $\begin{array}{l}\text { Donnerstag, 16. Juni 2005 } \\
\text { Bern }\end{array}$ & $\begin{array}{l}\text { Hotel } \\
\text { 9.30-16.30 Uhr }\end{array}$ & Bern \\
\hline \multirow{2}{*}{ Nr. 11 } & $\begin{array}{l}\text { Donnerstag, 15. September 2005 } \\
\text { Zürich }\end{array}$ & $\begin{array}{l}\text { FMT } \\
\text { 9.30-16.30 Uhr }\end{array}$ & Zürich \\
\hline
\end{tabular}

\section{Anmeldung}

Sie können sich bei nachfolgend aufgeführter Adresse (FMH Consulting Services) oder via Internet unter www.fmhservices.ch einschreiben.

\section{Praxiscomputerworkshop}

\section{Teilnehmende}

Der Workshop richtet sich an praxiseröffnende sowie an bereits praxistätige Ärztinnen und Ärzte.

\section{Inhalt}

Die Seminarteilnehmer/innen erhalten die Gelegenheit, die Praxisadministrationssoftware von sechs EDV-Unternehmen kennenzulernen. Sie erhalten einen Einblick in TARMED-kompatible Lösungen für die Leistungsabrechnung, die Führung elektronischer Krankengeschichten, die Einbindung von Laborgeräten und von Finanzbuchhaltungslösungen. Bei den anwesenden Firmen handelt es sich jeweils um fünf Hersteller von EDV-Gesamtlösungen in der Arztpraxis sowie um eine Abrechnungskasse. Zudem wird innerhalb des Einführungsreferates zum Thema «TrustCenter - was habe ich in meiner Praxis davon?» der Sinn und Zweck der TrustCenter sowie deren Nutzen für die einzelne Arztpraxis aufgezeigt.

\section{Ziel}

Die Workshopteilnehmer/innen erhalten einen ersten Überblick über führende Softwarelösungen. Sie erfahren, wie bei der Evaluation eines Ärztepaketes zweckmässigerweise vorgegangen wird und welche Fehler vermieden werden sollten.

\section{Kosten}

Der Workshop ist für FMH-Mitglieder gratis.

\section{Daten}

\begin{tabular}{|c|c|c|}
\hline \multirow[t]{2}{*}{ Nr. 14} & Donnerstag, 30. Juni 2005 & \multirow{2}{*}{$\begin{array}{l}\text { BEA Bern } \\
\text { Expo }\end{array}$} \\
\hline & Bern $\quad 14.00-17.30 \mathrm{Uhr}$ & \\
\hline & $\begin{array}{ll}\text { Donnerstag, } & 24 . \text { November } 2005 \\
\text { Zürich } & 14.00-17.30 \mathrm{Uhr}\end{array}$ & $\begin{array}{l}\text { Kongress- } \\
\text { haus }\end{array}$ \\
\hline
\end{tabular}

\section{Röntgen in der Arztpraxis}

\section{Teilnehmende}

Das Seminar richtet sich an Ärztinnen und Ärzte mit bestehender Praxis und an solche, die vor einer Praxiseröffnung oder Praxisübernahme stehen. 


\section{Themen}

Das Seminar vermittelt einerseits Kenntnisse über die gesamte bestehende Röntgenthematik, andererseits werden klare Trends für die Praxis der nächsten Generation mit digitalem Röntgen aufgezeigt. Als spezifische Themen werden behandelt: Röntgentarife nach TARMED, Rentabilität Röntgen in der Arztpraxis, bestehende und neue Vorschriften ab 1. Januar 2008 (kann mit einem Wechsel der Anlage verbunden sein; Achtung bei Praxisübernahmen). Zudem wird einerseits eine Checkliste vorgestellt und durchgearbeitet für die Evaluation und die Beschaffung einer neuen Anlage, andererseits werden Bewertungskriterien für die Übernahme einer Röntgenanlage (bei Praxisübernahmen) aufgezeigt. Die Seminarteilnehmer erstellen und bearbeiten innerhalb des Seminars digitale Röntgenbilder.

\section{Unterlagen}

Die Seminarteilnehmer/innen erhalten ein Skript mit Zahlen einer Arztpraxis mit Kostenberechnung, Amortisation und interessanten Leasingformen (ab welcher Auslastung arbeite ich kostendeckend oder mit Gewinn?). Zudem erhalten sie eine Checkliste für die Evaluation und die Beschaffung einer neuen Röntgenanlage sowie eine CD-ROM oder ein Fachbuch mit über 50 Modellaufnahmen.

\section{Kosten}

Fr. 300.- (inkl. sämtlicher Kursunterlagen und Verpflegung).

\section{Daten}

\begin{tabular}{|c|c|c|}
\hline Nr. 17 & $\begin{array}{l}\text { Donnerstag, } 19 . \text { Mai } 2005 \\
\text { Cham } \quad 9.30-16.00 \mathrm{Uhr}\end{array}$ & $\begin{array}{l}\text { Ausbildungs- } \\
\text { zentrum } \\
\text { Galexis }\end{array}$ \\
\hline Nr. 18 & $\begin{array}{ll}\text { Donnerstag, } & \text { 1. September } 2005 \\
\text { Burgdorf } & 9.30-16.00 \mathrm{Uhr}\end{array}$ & $\begin{array}{l}\text { Typon } \\
\text { Imaging AG }\end{array}$ \\
\hline Nr. 19 & $\begin{array}{l}\text { Donnerstag, } 27 . \text { Oktober } 2005 \\
\text { Cham } \quad 9.30-16.00 \mathrm{Uhr}\end{array}$ & $\begin{array}{l}\text { Ausbildungs- } \\
\text { zentrum } \\
\text { Galexis }\end{array}$ \\
\hline
\end{tabular}

\section{Anmeldung}

Das Seminar ist auf maximal 15 Personen beschränkt. Sie können sich bei nachfolgend aufgeführter Adresse (FMH Consulting Services) oder via Internet unter www.fmhservices.ch einschreiben.

\section{Anmeldung und Auskunft}

FMH Consulting Services, Simone Köpfli, Burghöhe 1, 6208 Oberkirch, Tel. 04192500 77, Fax 0419210586 oder via Internet unter www. fmhservices.ch.

\section{Hinweis}

Bei sämtlichen Seminaren, bei welchen die Kosten teilweise oder gänzlich von Seminarsponsoren gedeckt werden, werden die Teilnehmeradressen den jeweiligen Sponsoren zur Verfügung gestellt.

\section{Annullierungsbedingungen}

Bei den Gratisseminaren (exkl. Praxiscomputerworkshop) werden bei Abmeldungen oder Fernbleiben folgende Unkostenbeiträge erhoben:

- Fr. 50.- pro Person ab 14 Tage vor Seminarbeginn;

- Fr. 80.- pro Person ab 7 Tage vor Seminarbeginn oder Fernbleiben.

Bei Abmeldungen oder Fernbleiben bei kostenpflichtigen Seminaren werden die folgenden Unkostenbeiträge erhoben:

- $30 \%$ der Seminarkosten ab 14 Tage vor Seminarbeginn;

- $\quad 100 \%$ der Seminarkosten ab 7 Tage vor Seminarbeginn oder Fernbleiben. 